\title{
Dog Dander Antigen IgA Antibody Measurement
}

National Cancer Institute

\section{Source}

National Cancer Institute. Dog Dander Antigen IgA Antibody Measurement. NCI

Thesaurus. Code C130130.

A measurement of the dog dander antigen IgA antibody in a biological specimen. 